\title{
Region Based Feature Fusion of Imperfect Face and Gait Cues for human recognition using Median-LBPF and Median-LBPG based PCA followed by LDA
}

\author{
Annbuselvi K ${ }^{1}$, Santhi N ${ }^{2}$, Dr.S.Sivakumar ${ }^{3}$ \\ ${ }^{1}$ Associate Professor Department of Computer Science V.V.Vanniaperumal College for Women \\ Virudhunagar, India \\ ${ }^{2}$ Assistant Professor Department of Computer Science V.V.Vanniaperumal College for Women \\ Virudhunagar, India \\ ${ }^{3}$ Principal Cardamom Planters' Association College, Bodinayakanur, India
}

\begin{abstract}
:
Conventional computer-based multimodal biometric systems for human recognition based on face and gait cues are mainly based on recognition of perfect images of face and gait. There are situations, where perfect face and gait images may not be available which means probe images are imperfect. This paper proposes new methods Median Local Binary Pattern of Face image (Median-LBPF) and Gait image (Median-LBPG) to extract the features of imperfect face and gait images efficiently representing such imperfect images for better recognition. Initially the given imperfect face and gait images are divided into six overlapped regions called top, bottom, left, right, vertical center, horizontally center overlapped half images. The features of these six overlapped regions of imperfect face and gait images in the spatial domain are extracted by using MedianLBPF and Median-LBPG. Subsequently the dimensionality of the feature sets are reduced by a two stage feature reduction algorithms Principal Component Analysis (PCA) followed by Linear Discriminant Analysis (LDA). Next the individual face features and gait features are normalized to have their values lie within similar ranges and are concatenated at feature level. For classification, Euclidean distance measure is used to calculate the minimum of minimum distance between the six overlapped regions of given imperfect face and gait probe images and the corresponding regions of all six overlapped regions in the training sets. The proposed methods are tested by using publically available data sets ORL face and CASIA gait. The experimental results show that features of a region of face and gait images are adequate for recognition and its average recognition performance is same as perfect face and gait images
\end{abstract}

Keywords: Multimodal, Median-LBPF, Median-LBPG, PCA followed by LDA, Feature Fusion

\section{Introduction:}

Biometric based recognition is now a reliable technology that is being used for a variety of purposes such as surveillance system, security system, access control and etc. Different cues may be used for recognition based on the number of pixels presents on the individual. For optimal performance, the systemmay utilize as many cues as possible and combine them in significant ways [1]. There are two ways to fuse the informations. 1) Data fusion, where data can be combined and a decision can be reached based on the merged data. 2) Decision fusion, where each feature can be matched separately, perhaps using different methods, and then decisions taken can be merged.
Several multimodal strategies have been presented in recentyears that combine a physical biometric face with a behavioural biometric gait with the goal of seeing if combining the two will improve the performance of methods that just use one of these biometrics [2]. The primary reasons for combining the face and gait biometrics are that, because face is a short range biometric,it can only be successfully recognised when the individual is close enough to the camera for sufficient details of facialfeatures to be captured and gait is a medium to long range biometric, it can be reliably retrieved even from low resolutionimages and is less impacted by small 
changes in viewpoint. When these two biometric indicators are coupled, the system may be more resistant to changes in the distance between the person and the camera [3]. As such, multimodal human recognition is mainly carried out by perfect data of face and gait images. In reality, there are frequent situations where perfect face and gait images may not be available due to lighting changes, pose and partial occlusion. Hence, the problem of biometric system based on imperfect face and gait probes is still a new area of research.

Computer aided human recognition based on imperfect face or gait images is troubled, the works close to this issue appearsto be reasonably sparse and not so consistent. In current years some methods have been introduced to solve such problems.

In current years some methods have been introduced to solve such problems. In order to deal recognition of partial face expression, in [4] Charoenpong Theekapun et.al. proposed a displacement vector, a new method for recognising partial face expression from a $2.5 \mathrm{D}$ partial face image obtained from a viewpoint between $+/-45$ deg.

Yet in [5] Zheng-Hai Huang et.al. presented a face recognition technique using non-uniform patches via 2D-DWT, where for training and testing samples, a non-uniform patch technique for the top-lowfrequency level's sub-band is obtained and the nearest neighbour classifier and majority voting make the ultimate decision.

Further in [6] Lee K et.al. offered S-LNMF, a Selective Local Non-Negative Matrix Factorization method for occlusion based face recognition, where face images were divided into non-overlapping pieces and then PCA was employed on each piece to extract features, Next, a NN classifier was used to find misrepresentations in the subspace, whereas LNMF was used on non-occluded sections as a comparison and finally the match is found using Euclidean distance.

Yet in [7] Yumi Iwashita et.al. presented a method, where The human body image is broken into several pieces, then features of each pieces are retrieved. The similarity between the training templates and the retrieved features is then used to establish a matching weight for each piece, finally, a weighted integration of similarities in all components identifies the human.

Accurate human recognition using face and gait biometrics depend on high quality biometrics representations. A good biometric representation should discriminate the change in biometric identify. Conventional biometric representations are built on local descriptors, e.g., Local Binary Patterns (LBP) [8]. One of the most essential properties of the LBP is its resistance to monotonic gray-scale variations. LBP methodology has been developed recently with many variants [9] to increase performance in various applications. These variants focus on different features of the original LBP, such as (1) improving discriminative capabilities, (2) increasing robustness, (3) selecting its neighbourhood, and (4) combining with other techniques.

The LBP specifies a set of patterns that can be used to describe local structures. More patterns or information could be encoded to improve their discriminative capacity. In this paper we offered new methods Median Local Binary Pattern of Face image (MedianLBPF) and Gait image (Median-LBPG) to extract the discriminative features of imperfect face and imperfect gait cues. The local median is used as the threshold in Median-LBPF and Median-LBPG, rather than the grey value of the central pixel. The value of the centre pixel is also included, doubling the number of LBPF and LBPG.

Initially the given imperfect face and gait images are divided into six overlapped regions called top, bottom, left, right, vertical center, horizontally center overlapped half images. Next the local features of the six overlapped regions of imperfect face and gait images in the spatial domain are extracted by using proposed methods Median Local Binary Pattern of Face image (Median-LBPF) and Gait image (MedianLBPG). Subsequently the dimensionality of the feature sets of regions are reduced by a two stage feature reduction algorithms Principal Component Analysis (PCA) followed by Linear Discriminant Analysis (LDA) [10]. Next the individual face features and gait features are normalized to have their values lie within similar ranges and are concatenated at feature level. For classification, Euclidean distance measure is used to calculate the minimum of minimum distance between the six overlapped regions of given imperfect face and gait probe images and the corresponding regions of all six overlapped regions in the training sets.

The rest of the paper is organized as follows. Section II gives an overview of Local Binary Pattern (LBP), proposed methods Median Local Binary Pattern of Face image (Median-LBPF) and Gait image (MedianLBPG). Also provide a region based feature fusion of imperfect face and gait cues for human recognition 
using Median-LBPF and Median-LBPG based PCA followed by LDA. Section III presents results and discussion and finally, Section IV provides the conclusion.

\section{Materials and Methods:}

\section{A. Local Binary Pattern (LBP)}

In human identification, the Local Binary Pattern (LBP) has been effectively used as a local feature extraction method [8]. The LBP operator assigns decimal numbers to each pixel in a picture, called as LBP codes, which represent the local structure of each pixel. By subtracting the centre pixel value, each pixel is compared to its eight neighbours in a $3 \mathrm{X} 3$ neighbourhood. The strictly negative values are encoded with 0 whereas the others are encoded with 1 . Concatenating all of these binary codes in a clockwise direction, starting with the top-left one, generates a binary number, which is then labelled with the decimal value that corresponds to it. The derived binary numbers are referred to as LBP codes. It is formally defined as

$\operatorname{LBP}\left(x_{c}, y_{c}\right)=\sum_{i=0}^{7} \delta\left(p_{i}-p_{c}\right) 2^{i}$

where $p_{i}$ be the pixel value at $\mathrm{i}$ in a $3 \times 3$ neighborhoods of a centering pixel $p_{c}$ at $\left(x_{c}, y_{c}\right)$ and thresholding each pixel with the center pixel value using $\delta(x)$ where $x=p_{i}-p_{c}$.

$\delta(x)= \begin{cases}1 & \text { if } x \geq 0 \\ 0 & \text { if } x<0\end{cases}$

The computation of LBP is shown in Fig. 1 with the help of an example to make it easier to grasp.

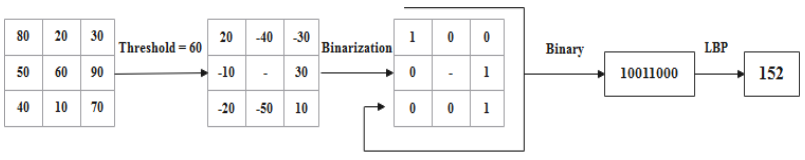

Fig.1 Illustration of computation of LBP

\section{B. Proposed Median Local Binary Pattern of Face image (Median-LBPF) and Gait image (Median- LBPG)}

The LBP approach has been recently developed with numerous variations to increase performance in various applications. These variations concentrate on various features of the original LBP operator, such as improving its discriminative capability, increasing its robustness, selecting its neighbourhood, and combining it with other methods.

More patterns or information could be encoded for extracting local structures to improve their discriminative capabilities. Median Local Binary Pattern of Face image (Median-LBPF) and Gait image (Median-LBPG) is variant of LBP to extract more local features under certain circumstances.

Median-LBPF and Median-LBPG should consider: (1) Illumination invariance, is the process of ensuring that texture indices have the same value even when the grey scale changes across the image. (2) Multi resolution invariance is a technique for recognising texture regardless of distance from an object. (3) Robustness, the strength against noise and other objects.

In Median-LBPF and Median-LBPG, the centering pixel value is calculated by using the median value of all 3 X 3 neighborhoods including center pixel at $\left(\mathrm{x}_{\mathrm{c}}, \mathrm{y}_{\mathrm{c}}\right)$. Let the median value $\mathrm{m}_{\mathrm{c}}$ is denoted as

$m_{c}=\operatorname{median}\left(p_{i}\right)$

where $i=0,1,2,3 \ldots 8$ the $3 \times 3$ neighborhood pixels $p_{i}$ including center pixel at $\left(x_{c}, y_{c}\right)$. Then, compare the median intensity of all the pixels in the $3 \times 3$ neighbourhood to all the pixels (including the centre pixel). The values that are strictly negative are encoded with 0 and the rest with 1 . A binary number is created by arranging all of these binary codes in a clockwise orientation, beginning with the top left pixel and ending with the centre pixel, with the matching decimal value used for labelling. The resulting binary numbers of face image is called Median-LBPF and gait image is called Median-LBPG. The Median-LBPF is formally defined as

Median $\operatorname{LBPF}\left(x_{c}, y_{c}\right)=\sum_{i=0}^{8} \delta\left(p_{i}-m_{c}\right) 2^{i}$

where $m_{c}$ is the median of pixel values in a $3 \times 3$ neighborhoods including center pixel, $p_{i}$ be the pixel value at $i$ in a $3 \times 3$ neighborhoods of a centering pixel $p_{c}$ at $\left(x_{c}, y_{c}\right)$ and thresholding each pixel with the center pixel value using $\delta(x)$ where $x=p_{i}-p_{c}$

$\delta(x)=\left\{\begin{array}{l}1, \text { if } x \geq 0 \\ 0, \text { if } x<0\end{array}\right.$

Fig. 2 shows the result of Median-LBPF for the same illustrative example.

For extracting local characteristics, the LBP gives a set of patterns. The discriminative capability is increased by feeding more patterns. The suggested MedianLBPF, in particular, compares all pixels in the region, including the central pixel, to the median intensity of all pixels in the region is shown in Fig. 2. 


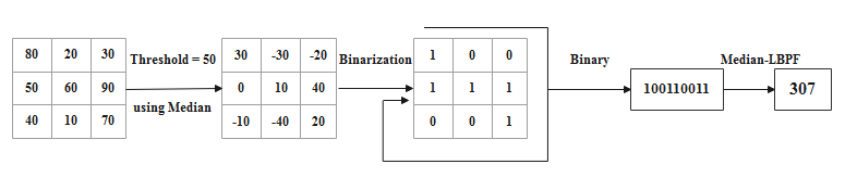

Fig.2 Illustration of computation of Median-LBPF

In a 3 X 3 neighbourhood, for example, the LBP produces only $256\left(2^{8}\right)$ patterns, whereas the Median-LBPF yields 511 patterns $\left(2^{9}-1\right)$ because all zero's and ones are the same. Fig. 3 shows a sample input face image obtained by using LBP and proposed Median-LBPF methods. Now, we apply such Median-LBPF and Median-LBPG operators over the six overlapped regions of imperfect face and gait images to extract the discriminative features, because each region of imperfect face and gait images may contain useful information for human recognition. Fig. 4 shows six overlapped Imperfect face image obtained by using LBP and proposed Median-LBPF methods.

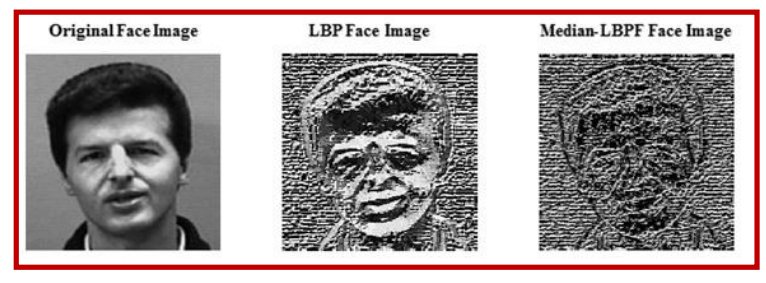

Fig.3 Sample input face image obtained by using LBP and Median-LBPF

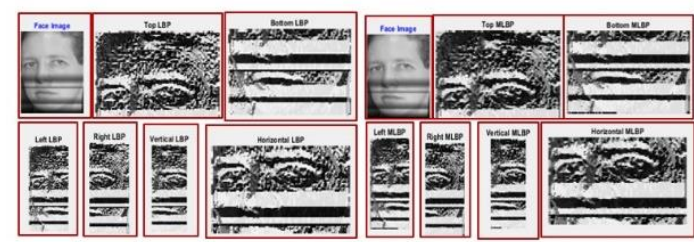

Fig.4 Six overlapped Imperfect face image obtained by using LBP and Median-LBPF

\section{Region based feature fusion of imperfect face and gait cues for human recognition using Median- LBPF and Median-LBPG based PCA followed by LDA}

The imperfect region based multimodal recognition comprises face and gait region partition, feature extraction using Median-LBPF and Median-LBPG, dimensionality reduction using PCA followed by LDA and finally classification using Euclidean distance. Initially the face and gait training data sets are restructured into six classes of top, bottom, left, right, vertical center and horizontal center, where the face and gait images in the data sets are partitioned into six overlapped half regions as top, bottom, left, right, vertical center, horizontal center and they are mapped into their corresponding classes. Next, the imperfect face and gait probe images are also partitioned into six overlapped half regions as top, bottom, left, right, vertical center and horizontal center.

To construct discriminative feature set of face and gait, first we apply the proposed methods Median-LBPF over face and Median-LBPG over gait by computing value for the centering pixel, using median value of all 3 X 3 neighborhoods plus center pixel using the formula given in section II. B equation (4) for effective feature representation.

Next a two stage PCA followed by LDA dimensionality reduction algorithms is applied to decrease the dimension of face and gait feature vectors [3][10]. In this the lower dimensional spaces produced by PCA which contains principal components of all images in the selected class to LDA which performs class separability and construct feature vectors for all classes in the restructured trained set.

The feature sets of face and gait are incompatible, so the individual face features and gait features are normalized to have their values lie within similar ranges and are concatenated at feature level to obtain a single feature vector. Next for classification, Euclidean distance measure is used to calculate the minimum of minimum distance between the six overlapped regions of given imperfect face and gait probe images and the corresponding regions of all six overlapped regions in the training sets.

The frame work of the method is given in Fig. 5 and Fig 6 and the procedure is given in Algorithm 1.

Algorithm 1: Integrating face and gait cues for multimodal human recognition at feature level

Input: Face Dataset, Gait Dataset, Imperfect face and gait test image

Output: Recognition result (Genuine/Imposter) i.e. (imperfect face and gait test images are recognized with train data set or not)

\section{Steps: Fusion at Feature Level:}

\section{Restructuring / Remodeling of training dataset}

Region wise partition of Face \& Gait Dataset:

1. Each face/gait image I with size $\mathrm{r} X \mathrm{c}$ in selected face dataset is partitioned into six overlapped regions as top, bottom, left, right, vertical center, horizontal center by using the following formula. top $=\mathrm{I}(1: \mathrm{r} / 2+10,1: \mathrm{c},:), \quad$ bottom $=\mathrm{I}(\mathrm{r} / 2-10: \mathrm{r}, 1: \mathrm{c},:)$, left $=\mathrm{I}(1: \mathrm{r}, 1: \mathrm{c} / 2+10,:), \quad$ right $=\mathrm{I}(1: \mathrm{r}, \mathrm{c} / 2-10: \mathrm{c},:)$, vertical center $=\mathrm{I}(1: \mathrm{r}, \mathrm{c} / 4:(3 / 4) * \mathrm{c},:)$, horizontal center $=\mathrm{I}(\mathrm{r} / 4:(3 / 4) * \mathrm{r}, 1: \mathrm{c},:)$ 
a) Face Data set: Formulate six classes of overlapped regions of face images as top $(\mathrm{F} 1), \quad$ bottom $(\mathrm{F} 2), \operatorname{left}(\mathrm{F} 3), \operatorname{right}(\mathrm{F} 4)$, vertical center(F5) and horizontal center(F6) where all top, bottom, left, right, vertical center, horizontal center regions are mapped into the corresponding top $(\mathrm{F} 1), \quad$ bottom $(\mathrm{F} 2), \operatorname{left}(\mathrm{F} 3), \operatorname{right}(\mathrm{F} 4)$, vertical center(F5) and horizontal center(F6) classes.

b) Gait Data Set: Formulate six classes of overlapped regions of gait images as top $(\mathrm{G} 1)$, bottom(G2), left(G3), $\operatorname{right}(\mathrm{G} 4)$, vertical center(G5) and horizontal center(G6) where all top, bottom, left, right, vertical center, horizontal center regions are mapped into the corresponding top $(\mathrm{G} 1)$, bottom(G2), left(G3), $\operatorname{right}(\mathrm{G} 4)$, vertical center(G5) and horizontal center(G6) classes.

2. Restructuring / Remodeling of given perfect/imperfect test image:

Region partition of imperfect Face \& Gait probe image.

a) Face Test Image: Partition the imperfect probe face images into six overlapped regions denoted as top(f1), bottom(f2), left(f3), right(f4), vertical center(f5) and horizontal center(f6).

b) Gait Silhouette Test Image: Partition the imperfect gait test images into six overlapped regions denoted as top(g1), bottom(g2), left(g3), right(g4), vertical center(g5) and horizontal center(g6).

3. Construction of Feature Vectors using MedianLBPF and PCA followed by LDA

\section{Training Phase:}

\section{Face Feature Vectors:}

a) Apply proposed Median-LBPF to each classes F1, F2, F3, F4, F5, F6 to extract the features sets and denoted as MLF1, MLF2, MLF3, MLF4, MLF5, MLF6.

b) Construct lower dimensional eigen face images M1, M2, M3, M4, M5, M6 for all images in MLF1, MLF2, ML3, MLF4, MLF5, MLF6 using conventional PCA procedure.

c) Construct eigen to fisher feature vectors FV1, FV2, FV3, FV4, FV5, FV6 for all images in the group of eigen face images M1, M2, M3, M4, M5, M6 using LDA.

\section{Gait Feature Vectors:}

a) Apply proposed Median-LBPF to each classes G1, G2, G3, G4, G5, G6 to extract the features sets and denoted as MLG1, MLG2, MLG3, MLG 4, MLG5, MLG6.

b) Construct lower dimensional eigen gait images M1, M2, M3, M4, M5, M6 for all images in MLG1, MLG2, MLG3, MLG4, MLG5, MLG6 using conventional PCA procedure.

c) Construct eigen to fisher feature vectors GV1, GV2, GV3, GV4, GV5, GV6 for all images in the classes of eigen gait images M1, M2, M3, M4, M5, M6 using LDA.

\section{Testing Phase:}

\section{Face Feature Vectors:}

a) Apply proposed Median-LBPG to the regions $\mathrm{f} 1, \mathrm{f} 2, \mathrm{f} 3, \mathrm{f} 4, \mathrm{f} 5, \mathrm{f} 6$ to extract the features sets and denoted as $\mathrm{mlg} 1, \mathrm{mlg} 2$, $\mathrm{mlg} 3$, mlg4, mlg5, mlg6.

b) Construct lower dimensional eigen images $\mathrm{m} 1, \mathrm{~m} 2, \mathrm{~m} 3, \mathrm{~m} 4, \mathrm{~m} 5, \mathrm{~m} 6$ for all $\mathrm{mlg} 1$, mlg2, mlg3, mlg4, mlg5, mlg6 imperfect face probe image using PCA

c) Construct eigen to fisher feature vectors $\mathrm{fv} 1, \mathrm{fv} 2, \mathrm{fv} 3, \mathrm{fv} 4, \mathrm{fv} 5, \mathrm{fv} 6$ for all eigen face images $\mathrm{m} 1, \mathrm{~m} 2, \mathrm{~m} 3, \mathrm{~m} 4, \mathrm{~m} 5, \mathrm{~m} 6$ using LDA.

\section{Gait Feature Vectors:}

d) Apply proposed Median-LBPG to the regions g1, g2, g3, g4, g5, g6 to extract the features sets and denoted as $\mathrm{mlg} 1, \mathrm{mlg} 2$, $\mathrm{mlg} 3$, mlg4, mlg5, mlg6.

e) Construct lower dimensional eigen images $\mathrm{m} 1, \mathrm{~m} 2, \mathrm{~m} 3, \mathrm{~m} 4, \mathrm{~m} 5, \mathrm{~m} 6$ for all $\mathrm{mlg} 1$, $\mathrm{mlg} 2$, mlg3, mlg4, mlg5, mlg6 imperfect face probe image using PCA.

f) Construct eigen to fisher feature vectors gv1, gv2, gv3, gv4, gv5, gv6 for all eigen face images $\mathrm{m} 1, \mathrm{~m} 2, \mathrm{~m} 3, \mathrm{~m} 4, \mathrm{~m} 5, \mathrm{~m} 6$ using LDA.

\section{Feature Vectors Normalization:}

Before face features and gait features are combined, the individual face features and gait features are normalized to have their values lie within similar ranges. We use a linear method which provides a normalization via the respective 


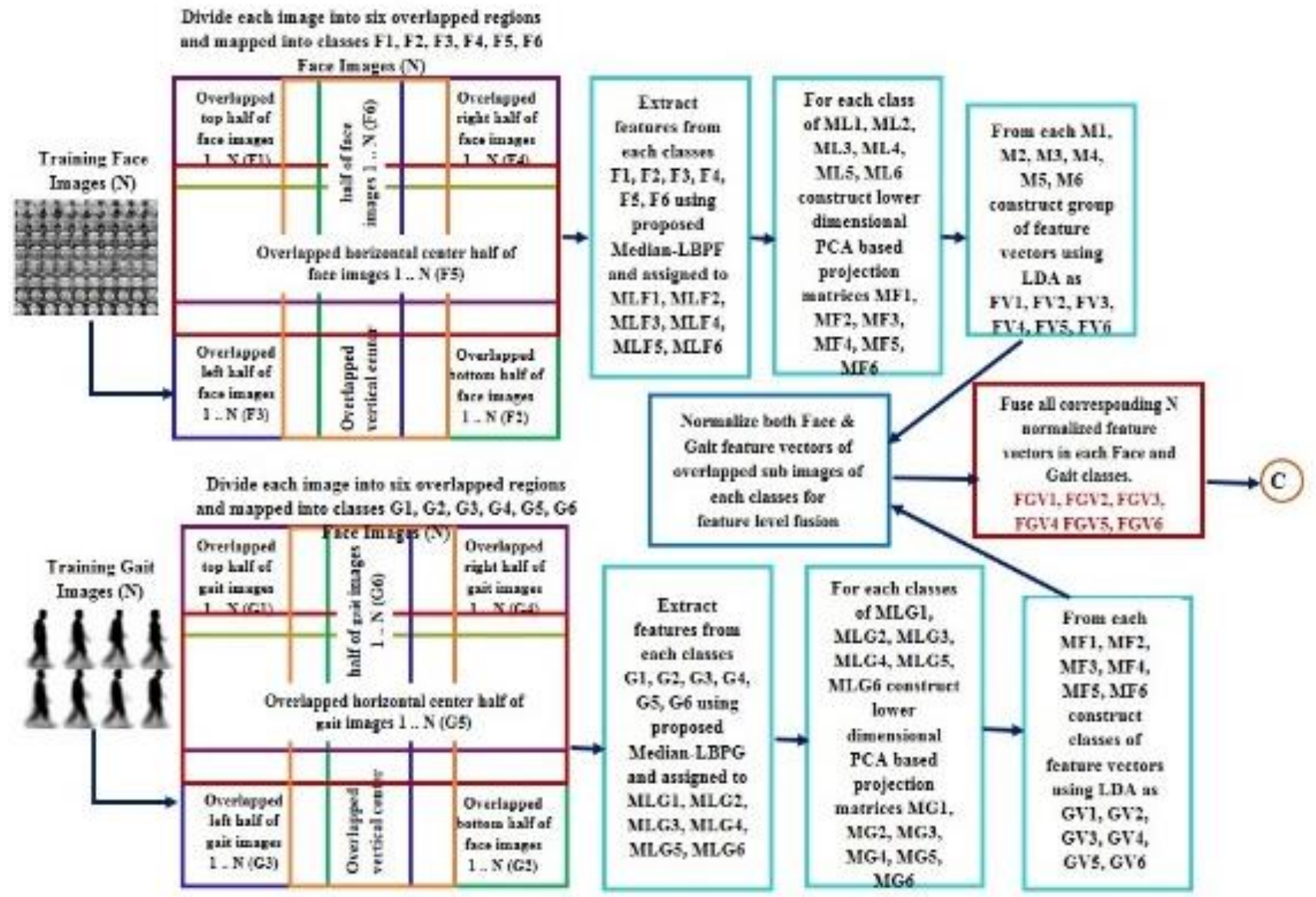

Fig. 5 Frame work of Feature Fusion of Training data sets Face and Gait using Median-LBPF and Median-LBPG based PCA followed by LDA

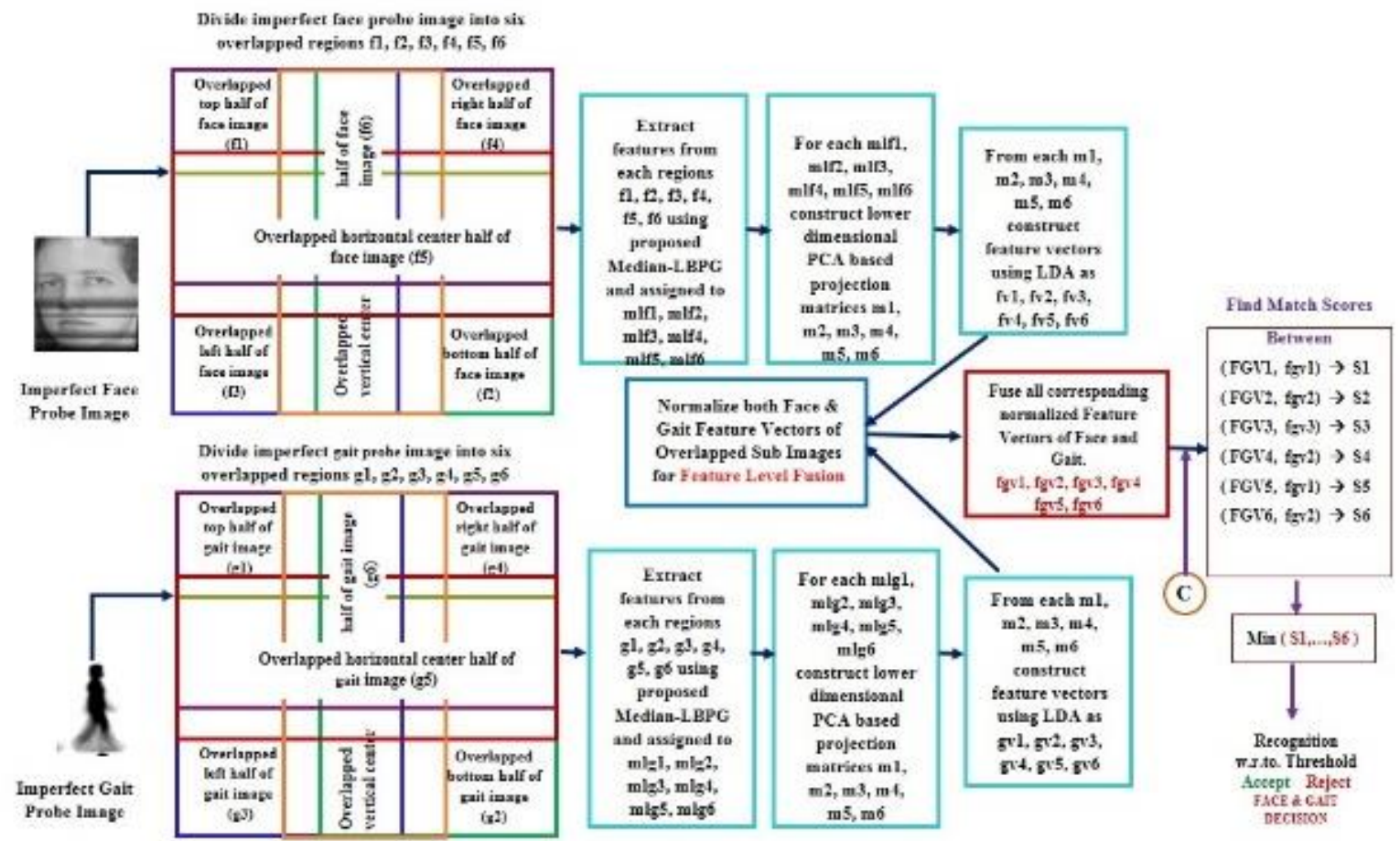

Fig. 6 Frame work of Imperfect Region based Feature Fusion of Face and Gait Cues for Human Recognition 
estimates of the mean and variance. The resulting normalized features have zero mean and unit variance.

Normalized training and testing Face Feature Vectors are: (FN1, FN2, FN3, FN4, FN5, FN6) and (fn1, fn2, fn3, fn4, fn5, fn6) resp.

Normalized training and testing Gait Feature Vectors are: (GN1, GN2, GN3, GN4, GN5, GN6) and (gn1, gn2, gn3, gn4, gn5, gn6) resp.

\section{Feature Fusion Phase :}

Fusing imperfect face and gait normalized feature vectors: The normalized imperfect face features and gait features are concatenated to form the features as

Training Phase: (FGV1, FGV2', FGV3', FGV4', FGV5', FGV6') = ([FN1,GN1], [FN2,GN2], [FN3,GN3], [FN4,GN4], [FN5,GN5], [FN6,GN6])

Testing Phase: (fgv1, fgv2, fgv3, fgv4, fgv5, fgv6) = ([fn1,gn1], [fn2,gn2], [fn3,gn3], [fn4,gn4], fn5,gn5], [fn6,gn6])

\section{Classification Phase :}

a) Calculating Match Score values: Apply Similarity / Distance Classifiers to find distance measures between normalized train and test feature vectors (FGV1', fgv1'), (FGV2, fgv2), (FGV3, fgv3), (FGV4, fgv4), (FGV5, fgv5) and (FGV6, fgv6) to get sets of (S1, S2, S3, S4, S5, S6)

b) Finding minimum score values of each set (S1, S2, S3, S4, S5, S6)

i) $\{\operatorname{Min} 1, \operatorname{Min} 2, \operatorname{Min} 3, \operatorname{Min} 4, \operatorname{Min} 5, \operatorname{Min} 6\}=$ $\{\min (\mathrm{S} 1), \quad \min (\mathrm{S} 2), \quad \min (\mathrm{S} 3), \quad \min (\mathrm{S} 4)$, $\min (\mathrm{S} 5), \min (\mathrm{S} 6)\}$

ii) find minimum value $-\operatorname{Min}=\min \{\operatorname{Min} 1$, Min2, Min3, Min4, Min5, Min6\}

\section{Recognition Phase :}

If Min value is less than the threshold value( $\eta$ ) the given imperfect face/gait test image is recognized as Genuine otherwise it is Imposter.

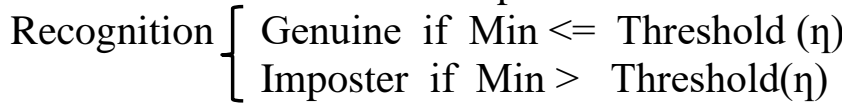

\section{Results and Discussion:}

To start this work, we have utilized face images from AT\&T "The Database of Faces" (formerly "The ORL Database of Faces") and gait from CASIA Database (Dataset B). For experimental part, we consider each face and gait training data sets with 50 genuine images corresponding to 10 subjects with five images per person and we set two testing data sets, one with imperfect probe face and gait images and other one with perfect face and gait images corresponding to 10 subjects with two images per person.

We present a comprehensive set of experiments using different parts of imperfect face and gait biometrics for human recognition. The method is tested with the two sets of data. The imperfect images were created solely with top half of the face, bottom half of the face, right half, left half, eyes and nose and etc.

Each image size in the face and gait data sets is 160X160. According to our proposed method each image is divided into six overlapped half regions as top, bottom, left, right, vertical center and horizontal center. So in training data sets after partition totally $50 \times 6=300$ images and are grouped into the six classes top, bottom, left, right, vertical center and horizontal center. Finally each class consists of 50 overlapped half images. Similarly in the two testing data sets after partition there are $20 \times 6=120$ images and are grouped into six classes top, bottom, left, right, vertical center and horizontal center. Finally each class in the testing data sets consist of 20 overlapped half images.

The imperfect face and gait test image features in the spatial domain are extracted by using proposed Median-LBPF and Median-LBPG. In a 3X3 neighbourhood the LBP produces only $256\left(2^{8}\right)$ patterns, whereas the Median-LBPF yields 511 patterns $\left(2^{9}-1\right)$. The suggested Median-LBPF, in particular, compares all pixels in the region, including the central pixel, to the median intensity of all pixels in the region. We applied such Median-LBPF and Median-LBPG operators over the six overlapped regions of imperfect face and gait images to extract the discriminative features.

Subsequently the dimensionality of the feature set is reduced by using a two stage PCA followed by LDA methods. The individual face features and gait features are normalized to have their values lie within similar ranges and are concatenated at feature level. 
Table 1. Comparison of Recognition rate using Perfect and Imperfect Face Biometric

\begin{tabular}{|l|c|c|c|c|c|c|c|}
\hline \multicolumn{2}{|c|}{ Table 1. Comparison of Recognition rate using Perfect and Imperfect Face Biometric } \\
\cline { 2 - 9 } \multicolumn{2}{|c|}{ Biometric Cue \& Method } & \multicolumn{7}{c|}{ top } & bottom & left & right & $\begin{array}{c}\text { vertical } \\
\text { center }\end{array}$ & $\begin{array}{c}\text { horizontal } \\
\text { center }\end{array}$ & $\begin{array}{c}\text { full } \\
\text { Perfect Face (LBP + PCA followed by LDA) }\end{array}$ & 72 & 75 & 71 & 70 & 76 & 74 & 82 \\
\hline $\begin{array}{l}\text { Perfect Face (Median LBPF + PCA followed by } \\
\text { LDA) }\end{array}$ & 80 & 82 & 79 & 78 & 83 & 80 & 84 \\
\hline Imperfect Face (LBP + PCA followed by LDA) & 71 & 73 & 69 & 70 & 74 & 72 & 80 \\
\hline $\begin{array}{l}\text { Imperfect Face (Proposed Median LBPF + PCA } \\
\text { followed by LDA) }\end{array}$ & 76 & 79 & 72 & 71 & 82 & 77 & 82 \\
\hline
\end{tabular}

\begin{tabular}{|c|c|c|c|c|c|c|c|}
\hline \multirow[b]{2}{*}{ Biometric Cue \& Method } & \multicolumn{7}{|c|}{ Recognition Rate $(\%)$} \\
\hline & top & bottom & left & right & $\begin{array}{l}\text { vertical } \\
\text { center }\end{array}$ & $\begin{array}{c}\text { horizontal } \\
\text { center }\end{array}$ & full \\
\hline Perfect Gait (LBP + PCA followed by LDA) & 69 & 75 & 71 & 69 & 73 & 71 & 80 \\
\hline $\begin{array}{l}\text { Perfect Gait (Median LBPG + PCA followed by } \\
\text { LDA) }\end{array}$ & 76 & 80 & 76 & 72 & 80 & 73 & 81 \\
\hline Imperfect Gait (LBP + PCA followed by LDA) & 66 & 73 & 69 & 69 & 72 & 70 & 78 \\
\hline $\begin{array}{l}\text { Imperfect Gait ( Proposed Median LBPG + PCA } \\
\text { followed by LDA) }\end{array}$ & 72 & 74 & 71 & 69 & 77 & 72 & 80 \\
\hline
\end{tabular}

\begin{tabular}{|c|c|c|c|c|c|c|c|}
\hline \multirow[b]{2}{*}{ Biometric Cues \& Method } & \multicolumn{7}{|c|}{ Recognition Rate $(\%)$} \\
\hline & top & bottom & left & right & $\begin{array}{l}\text { vertical } \\
\text { center }\end{array}$ & $\begin{array}{c}\text { horizontal } \\
\text { center }\end{array}$ & ful \\
\hline $\begin{array}{l}\text { Perfect Face + Gait (LBP + PCA followed by } \\
\text { LDA) }\end{array}$ & 80 & 78 & 72 & 70 & 74 & 72 & 87 \\
\hline $\begin{array}{l}\text { Perfect Face + Gait (Median LBPF +Median } \\
\text { LBPG + PCA followed by LDA) }\end{array}$ & 82 & 78 & 76 & 73 & 77 & 75 & 90 \\
\hline $\begin{array}{l}\text { Imperfect Face + Gait (LBP + PCA followed by } \\
\text { LDA) }\end{array}$ & 78 & 70 & 65 & 66 & 70 & 63 & 82 \\
\hline $\begin{array}{l}\text { Imperfect Face + Gait ( Proposed Median LBPF } \\
+ \text { Median LBPG + PCA followed by LDA) }\end{array}$ & 77 & 77 & 70 & 71 & 82 & 75 & 84 \\
\hline
\end{tabular}

\begin{tabular}{|c|c|c|c|c|c|c|c|}
\hline \multirow[b]{2}{*}{ Biometric Cues \& Method } & \multicolumn{7}{|c|}{ Recognition Rate (\%) } \\
\hline & top & bottom & left & right & $\begin{array}{l}\text { vertical } \\
\text { center }\end{array}$ & $\begin{array}{c}\text { horizontal } \\
\text { center }\end{array}$ & full \\
\hline $\begin{array}{l}\text { Imperfect Face (Median LBPF + PCA followed by } \\
\text { LDA) }\end{array}$ & 76 & 79 & 72 & 71 & 82 & 77 & 82 \\
\hline $\begin{array}{l}\text { Imperfect Gait (Median LBPG + PCA followed by } \\
\text { LDA) }\end{array}$ & 72 & 74 & 71 & 69 & 77 & 72 & 80 \\
\hline $\begin{array}{l}\text { Imperfect Face + Gait (Proposed Median LBPF + } \\
\text { Median LBPG + PCA followed by LDA) }\end{array}$ & 77 & 77 & 70 & 71 & 82 & 75 & 84 \\
\hline
\end{tabular}

For classification, Euclidean distance measure is used to calculate the minimum of minimum distance between the six overlapped regions of given imperfect face and gait probe images and the corresponding regions of all six overlapped regions in the training sets.

The proposed method's performance is evaluated in terms of recognition rate. The results are compared between unimodal and multimodal systems. The recognition rates using face biometric is given in table 1 and graphically presented in Fig. 7. Similarly table 2 shows gait biometric and pictorially shown in Fig. 8.
Likewise table 3 and table 4 show recognition rates of multimodal classifier and its comparison with unimodal at feature level respectively and visually presented in Fig. 9 and Fig.10.

As it can be inferred from the graph, multimodal classifier fused at feature level out performs well with recognition rate of $84 \%$. When probe images are imperfect the recognition rates of the proposed method is near to perfect probe images. The proposed method's performance in terms of recognition accuracy is measured and compared to some recent approaches [11]. 


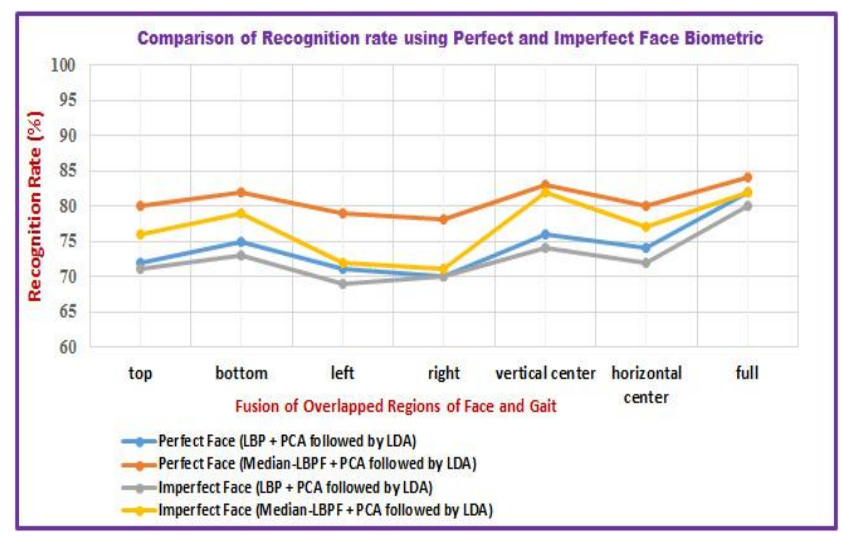

Fig. 7. Comparison of Recognition rate using Perfect and Imperfect Face Biometric

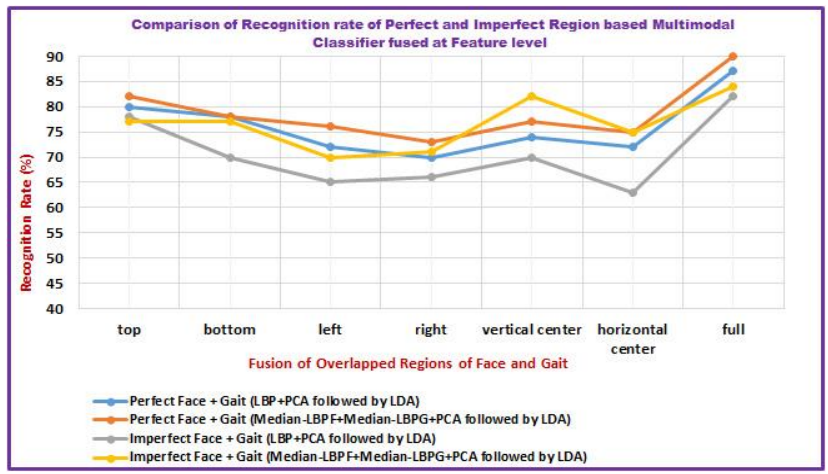

Fig. 9. Comparison of Recognition rate using Perfect and Imperfect Region based Multimodal Classifier fused at Feature Level

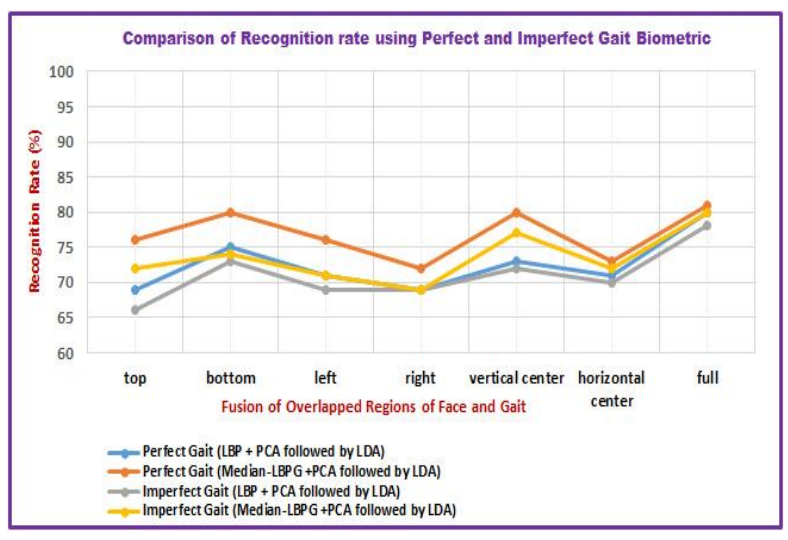

Fig. 8. Comparison of Recognition rate using Perfect and Imperfect Gait Biometric

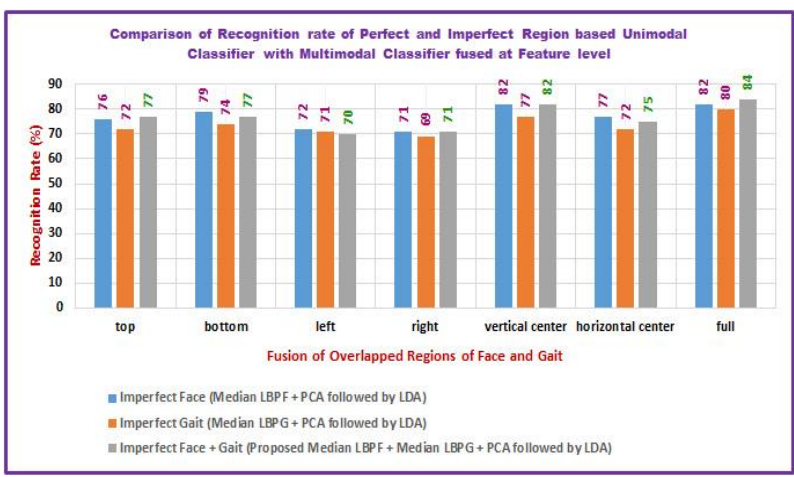

Fig. 10. Comparison of Recognition rate using Perfect and Imperfect Region based Unimodal Classifier with Multimodal Classifier fused at Feature Level

Next for classification, Euclidean distance measure is used to calculate the minimum of minimum distance between the six overlapped regions of given imperfect face and gait probe images and the corresponding regions of all six overlapped regions in the training sets. Outcomes are measured by using recognition rate. The results prove that overlapped half of a region of imperfect face and gait probe images are enough for recognition.

\section{References:}

[1] Annbuselvi.K, Santhi.N, Intelligences of Fusing Face and Gait in Multimodal Biometric System: A Contemporary Study, International Journal of Computer Science Trends and Technology (IJCST) - Volume X Issue X, ISSN: 2347-8578.

[2] Muhtahir O.Oloyede, and Gerhard P. Hancke, Unimodal and Multimodal Biometric Sensing Systems: A Review, IEEE, 2016. gait features are normalized to have their values lie within similar ranges and are concatenated at feature level to obtain a single feature vector. 
[3] Annbuselvi.K, Santhi.N, Role of Feature Extraction Techniques: PCA and LDA for Appearance Based Gait Recognition, International Journal of Computer Sciences and Engineering, Vol-6, Special Issue- 4, 2018, E-ISSN: 2347-2693.

[4] Charoenpong Theekapun, Shogo Tokai, Hiroyuki Hase, Facial Expression Recognition from a Partial Face Image by Using Displacement Vector, IEEE, Proceedings of ECTI-CON 2008.

[5] Zheng-Hai Huang a,c, Wen-Juan Li a, Jin Shanga,b, Jun Wang b, Ting Zhanga, Nonuniform patch based face recognition via 2DDWT, Elsevier, Image and Vision Computing 37 (2015) 12-19.

[6] Oh H., Lee K., and Lee S., "Occlusion Invariant Face Recognition Using Selective Local NonNegative Matrix Factorization Basis Images," Image and Vision Computing, 2008.

[7] Yumi Iwashita, Koji Uchino and Ryo Kurazume, Gait-Based Person Identification Robust to Changes in Appearance, Sensors 2013,13,7884-7901;doi : 10.3390/s130607884.
[8] Di Huang, Caifeng Shan, Mohsen Ardebilian, Yunhong Wang, and Liming Chen, Local Binary Patterns and Its Application to Facial Image Analysis: A Survey, IEEE Transactions on Systems, San, and Cybernetics - Part c: Applications and Reviews, vol. 41, no. 6, November 2011.

[9] Li Liu, Paul Fieguth, Xiaogang Wang, Matti Pietikainen, and Dewen Hu, Evaluation of LBP and Deep Texture Descriptors with a New Robustness Benchmark, Springer International Publishing AG 2016, pp. 69-86, DOI: 10.1007/978-3-319-46487-9 5.

[10] Santhi.N, Annbuselvi.K, Performance Analysis of Feature Extraction, International Journal of Engineering Research in Computer Science and Engineering, Vol 5, Issue 3, March 2018, ISSN (Online) 2394-2320.

[11] Ali Elmahmudi, Hassan Ugail, Deep face recognition using imperfect facial data, Elsevier, Future Generation Computer Systems 99 (2019) 213-225. 